\title{
Parafoveal word perception: A case against semantic preprocessing
}

\author{
ALBRECHT WERNER INHOFF and KEITH RAYNER \\ University of Massachusetts, Amherst, Massachusetts 01003
}

\begin{abstract}
The present experiment investigated semantic information extraction in parafoveal word perception. An ambiguous word (bank) was presented in foveal vision, and simultaneously a disambiguating word (water, money) was presented in the parafovea. Subjects were required to make a forced choice between two phrases, and the task was constructed so that a correct choice could be made if semantic information about both the foveal and parafoveal word had been obtained. However, the results indicated that the forced-choice results could be explained by two factors: identification of the parafoveal word and correct guessing. Hence, it was concluded that those models of reading which rely on unconscious semantic preprocessing of parafoveal words were not supported.
\end{abstract}

As early as 1898, Erdmann and Dodge (cited in Huey, 1908) observed that a reader gains partial information from parafoveal vision during an eye fixation in reading. However, the role and specification of parafoveal information in reading has remained controversial as reflected by different theoretical positions. Neisser's (1967) analysis-by-synthesis model and Hochberg's (1970) model of reading both rely on some form of semantic preprocessing in parafoveal vision. Neisser assumed that the reader anticipates meaning via parafoveal vision and subsequently confirms his hypothesis during the following foveal evaluation. In Hochberg's model, the reader uses parafoveal vision to determine where to find optimum information during a subsequent fixation (cognitive search guidance).

On the other hand, there is no corresponding semantic preprocessing of parafoveal information in a process-monitoring model of reading (McConkie, 1979; Rayner, 1979). In this model, semantic information is directly accessed for the area fixated; here, the reader tries to identify words as far to the right on a line of text as possible and the subsequent fixation position will be located at the boundary of the preceding area of word identification.

Bradshaw (1974) obtained results which support the semantic preprocessing position. A homograph was presented foveally and a disambiguating word

This research was supported by Grant HD12727 from the National Institute of Child Health and Human Development and Grant BNS79-17600 from the National Science Foundation. The first author was supported by a fellowship from the DAAD of the Federal Republic of Germany. We would like to thank Jerome L. Myers, Robert E. Morrison, Rex Bradford, Charles Clifton, and two anonymous reviewers for their comments and help. Requests for reprints should be sent to Albrecht Werner Inhoff, Department of Psychology, University of Massachusetts, Amherst, Massachusetts 01003 . was presented simultaneously in the parafovea. In a subsequent forced-choiced task, subjects biased their choices of homographic interpretation in the direction of the parafoveal word. This result held for parafoveal words that were reportable and also for those words the reader failed to identify. However, it should be pointed out that the effect of semantic preprocessing was marginal. Out of a total of $450 \mathrm{tri}-$ als in which the parafoveal word was not correctly reported, a biased choice based on parafoveal information was made $53 \%$ of the time. From this, Bradshaw concluded that semantic preprocessing represented the initial stage of parafoveal word perception. Allport (1977) and Marcel (1978) suggested a form of unconscious semantic preprocessing to account for their findings. In these studies, a masking paradigm was used to limit the availability of one of two words that were presented simultaneously so that only the unmasked word could be reported. In this condition, association strength between the masked and unmasked word affected the subjects' naming response of the unmasked word.

However, in the Allport (1977) and Marcel (1978) studies, both words were presented around the point of fixation; hence, there was no foveal and parafoveal distinction, and in Bradshaw's study (1974) neither was central fixation position controlled nor were different eccentricities investigated. Furthermore, the Bradshaw (1974) study is open to alternative interpretations and the findings do not necessarily demonstrate that the unidentified parafoveal word was processed up to a semantic level. Bradshaw displayed the stimuli for $125 \mathrm{msec}$, followed immediately by two different meanings of the homograph. Since a mask was not used, it may have been possible for the reader to compare the forced-choice alternatives with a still available iconic representation of the preceding parafoveal word. Also, since the parafoveal word and the 
following correct forced-choice alternatives may have been identical, the subject may have relied in the forced choice on a mere visual pattern match. Thus, it was not necessary to report or to identify the parafoveal word in this experiment in order to make a correct choice.

McConkie and Rayner (1975) and Rayner (1975) carefully controlled fixation position and used an experimental technique that allowed a display change during the reader's eye movement. They found that semantic information affected the reader's eye behavior only in the area of the fixation position and in the area immediately to its right, while for words presented 2 to $4 \mathrm{deg}$ of visual angle in the right visual field, initial letter, word shape, and word length information were effective. Rayner and Bertera (1979) masked the reader's foveal vision on each eye fixation and found no evidence for semantic preprocessing of the parafoveally available text. Subjects tended to report visually similar words, while errors preserving the semantic content were rare. Furthermore, the semantic preprocessing position is not consistent with findings reported by Rayner, McConkie, and Zola (1980). In that study, subjects were instructed to move their eyes from a previously blank space to a parafoveally presented word. During the eye movement, the initially displayed word was replaced by another word; the relationship between the two successive words was controlled. In the semantic relation condition, no effects were observed, while congruence of word length and initial letter information between the pre- and postsaccadic word resulted in faster naming performance.

However, the McConkie and Rayner (1975) and Rayner (1975) studies do not allow a distinction between the effects of semantic preprocessing and accomplished word identification. In the foveal masking experiment (Rayner \& Bertera, 1979) and the parafoveal replacement study (Rayner et al., 1980), a foveal word was not available, and this may have altered the parafoveal stimulus evaluation. Hence, a direct comparison of these studies with the experiments which support the semantic preprocessing position is not possible.

The present study was designed to be sensitive to semantic preprocessing in an experimental situation in which fixation location was controlled and different parafoveal locations were examined $(1,3$, and $5 \mathrm{deg}$ of visual angle) in both visual fields (to the right and left of fixation position). A foveal (center) word and a parafoveal (off-center) word were presented and assumed to allow a correct choice in a subsequent forced-choice task for those instances in which some semantic information of the parafoveal word had been acquired. Subjects were also asked to report the center and off-center words to determine if correct judgments for those trials in which the parafoveal word was not reported were due to semantic preprocessing. It was expected that, in the case of semantic preprocessing, subjects would perform better than chance in the forced-choice task even if the respective parafoveal word was not correctly identified and reported. Furthermore, two kinds of foveal stimuli, homographs and nonhomographs, were used to control for processing characteristics of different word types.

\section{METHOD}

\section{Subjects}

Eighteen students at the University of Massachusetts were paid or given course credit for their participation. All had normal or corrected to normal vision.

\section{Apparatus}

A cathode-ray tube (CRT) and two response panels were interfaced with a Hewlett Packard 2100 computer, which controlled the experiment. One response panel was used by the experimenter to initiate the experimental trials, while the second panel was used by the subject to make a forced-choice response.

The stimuli were presented in upper case on a Hewlett Packard 1300A CRT. The CRT has a P-31 phosphor, with the characteristic that removing a character results in a drop to $1 \%$ of maximum brightness in $.25 \mathrm{msec}$. Each letter was made up of dots from a 5 by 7 matrix. A black theater gel covered the screen, so that the stimuli appeared clear and sharp. A visual mask, used to cover the foveal and parafoveal word, matched the masked words in length and consisted of a homogeneously illuminated field.

During the experiment, the subjects' eyes were $46 \mathrm{~cm}$ from the CRT so that three character spaces equaled 1 deg of visual angle. The room was illuminated, and the luminance of the CRT held constant throughout the experiment. The computer recorded the subject's forced-choice response and, additionally, kept a record of the RT. This measure was the time interval between the presentation of the forced-choice alternatives and the subject's buttonpress response.

\section{Design and Material}

Seventy-two homographs (four- and five-letter words) with about equal probabilities for two different meanings were selected from Cramer (1970) and Webster's New Collegiate Dictionary (1973). Seventy-two nonambiguous words were matched for word frequency (Kučera \& Francis, 1967), word length, and number of syllables.

For each of these 144 words (called foveal, or center, words), two modifying words (called parafoveal, or off-center, words) were selected. In the case of homographs, each off-center modifier referred to a different meaning. For those homographs with association norms available (Cramer, 1970), the two selected biasing off-center words were matched for associative strength. For the remaining homographs, two off-center modifiers were selected which unambiguously referred to two specific meanings. Thus, the center homograph BANK was paired with the off-center word WATER or MONEY. For the nonhomographic controls, two off-center modifications were chosen which represented two different situations. Thus, for example, the center nonhomographic control WOMAN was paired with either RICH or POOR. In another control condition, the homographic and nonhomographic words were presented in the center location and the off-center word consisted of a repetition of the center word. Thus, in this condition, BANK was paired with BANK and WOMAN was paired with WOMAN. Those trials in which the center word was different from the off-center specification were termed biased, while the neutral trials in which the same word was presented 
twice were referred to as a pattern match. During each experimental trial, only one of the three possible off-center words was paired with the respective center word. Word length of the offcenter word ranged from three to six letters. Median word-frequency values were 96 for the off-center words biasing a central homograph and 86 for the nonhomographic modifiers. Each off-center word could be presented beginning 1,3 , or $5 \mathrm{deg}$ of visual angle to the right of fixation or ending 1,3 , or 5 deg to the left of fixation. Hence, for each center word, 18 different display conditions were possible, produced by factorially combining 3 off-center conditions by 3 visual angles by 2 visual fields. Order of homographic and nonhomographic center words, order of visual angle, and type of off-center word were randomized for a single subject but counterbalanced across the experimental population.

For the subsequent forced-choice task, two phrases were constructed. These phrases were short so as to minimize storage processes during the forced-choice task. They ranged from two to four words, with less than 32 character spaces each. Each phrase referred to a specific meaning of the homograph or depicted two different situations for the nonhomographic controls. Part of each phrase (the center word) was never spelled out. Its respective position in the two forced-choice phrases was indicated by three spaced dots. Thus, the forced-choice alternatives for the word WOMAN were A WEALTHY ... and A MONEYLESS .... The offcenter word was not repeated in the two forced-choice phrases. Rather, a word semantically related to the off-center specification was used in the correct forced-choice alternative to prevent simple pattern matching of a parafoveal word with the critical word in the correct phrase. It was assumed that the processing of the semantic relation between the parafoveal specification and the subsequent correct phrase allowed for a correct choice.

\section{Procedure}

Subjects participated individually and received detailed instructions at the beginning of an experimental session. Part of the instructions were 20 training trials, consisting of 10 homographic and 10 nonhomographic trials presented on sheets of paper. The importance of correct forced-choice performance based on the center and off-center word-pair evaluation was emphasized, while choice of either of the two forced-choice alternatives was optional in two pattern-match trials. The subject was given feedback about the training performance, and the experimenter tried to outline the desired inference processes for those trials in which an incorrect phrase was chosen. A particular trial is illustrated in Table 1. The subject was told that the cross on the top of the page represented fixation position during the experimental computercontrolled trials and that the top-down arrangement on the sheet of paper was analogous to the later temporal sequencing of an experimental trial.

The computer-controlled trials consisted of 6 additional practice trials on the CRT and the 144 experimental trials, the data from which were statistically analyzed. During the computercontrolled trials, the subject's head was stabilized with a foreheadrest to hold viewing distance constant. The subject was instructed to fixate a cross displayed in the center of the screen at the beginning of each trial and to indicate readiness, that is, successful fix- ation. Contingent on the subject's signal, the experimenter initiated the display of the center and off-center words, which were projected on the CRT for $150 \mathrm{msec}$ and immediately followed by a 50-msec brightness mask that covered the preceding center and off-center word completely. The choice of the display interval was determined so that the subject was not able to saccade successfully to the off-center word position while it was still displayed. At the same time, the display interval approximated the lower range of fixation durations in reading (Rayner, 1978). The visual mask that followed the word pair was used in order to control for iconic readout. After the mask, the screen remained blank for approximately $1 \mathrm{sec}$. Then two phrases were presented 2 and $2.5 \mathrm{~cm}$ below the initial fixation position. The instructions had reinforced an intuitive judgment for those trials in which the subject failed to identify both words of the word pair; again, choice was optional for the pattern-match trials. Phrase choice was indicated by a buttonpress response. The computer recorded the subject's forced-choice response and RT. The choice resulted in the erasure of the two forced-choice alternatives, and another two phrases were displayed at the same positions within the following $500 \mathrm{msec}$. The subject's task was then to report the center and offcenter word. The verbal response was recorded by the experimenter. Guessing and partial report were encouraged.

The forced-choice task preceded the report task in this experiment to keep the choice sensitive to intuitive judgments for those trials in which the subject failed to identify the off-center bias. An experimental session lasted 50 to $80 \mathrm{~min}$. Choice data were obtained for each of the 18 subjects; for reasons unrelated to the experiment, no report data were available for 2 of the subjects.

\section{RESULTS}

The data subjected to statistical evaluation were percent correct report of the parafoveal words, percent correct forced-choice performance, and RT values of the forced-choice responses.

\section{Correct Report}

The correct report of the parafoveal word, averaged across subjects, was $59 \%, 25 \%$, and $7.5 \%$ for the 1-, 3-, and 5-deg positions, respectively. Subjects had a higher probability to be correct for words displayed in the right parafoveal field $(39 \%)$ than for words displayed in the left field $(22 \%)$, and more parafoveal words were correctly reported in the pattern-match condition (36\%) than in either the homographic or the nonhomographic parafoveal bias $(27.5 \%$ and $28.5 \%$, respectively). Figure 1 shows the data.

A 3 (visual angle) by 2 (visual field) by 3 (modification: homographic, nonhomographic, or pattern

Table 1

A Simulated Trial as Provided in the Instruction

\begin{tabular}{lll}
\hline \multicolumn{1}{c}{ Center } & Off-Center & \multicolumn{1}{c}{ Temporal Sequence } \\
\hline $\mathrm{X}$ & & Fixation Position \\
CAR & BORROW & Word Pair (Nonhomographic) \\
$\ldots$ RENTAL & & Forced-Choice Alternatives \\
THE CCIDENT & & Word-Report Instruction \\
THE OFF-CENTER WORD WAS ... & & \\
\hline
\end{tabular}




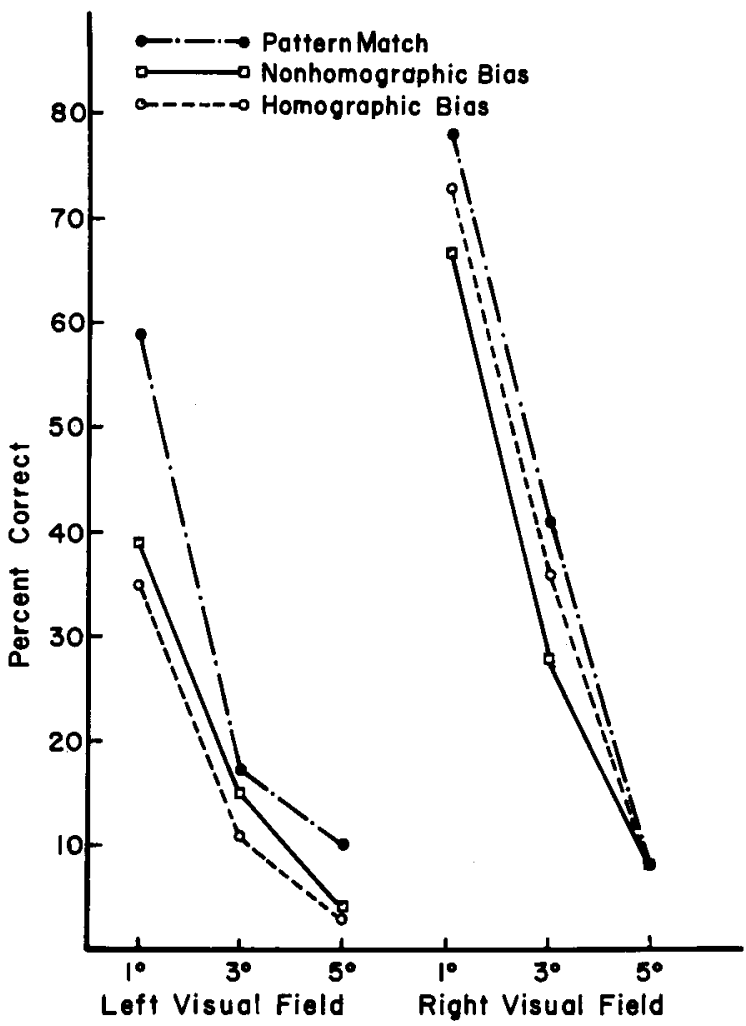

Figure 1. Percent correct report of parafoveal words.

match) ANOVA was performed on the correct-report data. All of the main effects were significant: visual angle $[F(2,30)=173.82, p<.001]$, visual field $[F(1,15)=40.68, \quad p<.001]$, and modification $[F(2,30)=3.76, p<.05]$. The interaction between visual angle and visual field was also highly significant $[F(2,30)=18.45, p<.001]$. That is, the difference in correctly reported parafoveal words between the right and left visual fields decreased with increasing visual angle. A Newman-Keuls test on the main effects indicated that each visual angle position differed from the two remaining locations $(p<.01)$. There was no difference between the two parafoveal bias conditions (homographic and nonhomographic), while both biasing conditions differed significantly $(p<.05)$ from the pattern-match condition.

Errors and partial reports were evaluated separately. Errors in reporting the foveal (center) word were rare, averaging about $1 \%$, and exceeded in no case more than $3 \%$ of the experimental trials. There was no instance of a partial foveal word report. Also, errors due to confusions of foveal and parafoveal word location, that is, reversal of word locations, were rare and amounted to less than $1 \%$ of those instances in which both foveal and parafoveal words were reported (though subjects indicated occasionally that their choices of word position were more like guessing). Error and partial report responses of the parafoveal word occurred on $32 \%$ of the experimental trials. Since the data analysis showed no difference, homographic and nonhomographic bias trials were pooled together. A report in which the parafoveal word was substituted with a semantically similar word was taken as evidence for semantic preprocessing, while a response containing word length or initial letter information of the original word was viewed as support of visual processes. Only $50 \%$ of the error and partial report responses could be classified according to the criteria; out of these, $15 \%$ were in accordance with the semantic preprocessing position and $85 \%$ favored visual representation.

The drastic difference in error and overall report performance of the parafoveal and foveal words demonstrates a successful foveal-parafoveal distinction across all experimental trials. Since both words were available only during the initial presentation of $150 \mathrm{msec}$, attention seems to have been focused on the center word.

\section{Forced-Choice Data}

An inspection of the forced-choice data revealed results that are analogous to the report performance. For the biased trials, correct choices were $73 \%, 59 \%$, and $56 \%$ at the 1-, 3-, and 5-deg positions, respectively. Performance in the right visual field exceeded performance in the left ( $66 \%$ vs. $60 \%$ ), while correct choices were equivalent for the homographic and nonhomographic parafoveal bias (63\% in both cases). Figure 2 shows the forced-choice data. A 2 (homographic and nonhomographic bias) by 2 (phrase location) by 2 (visual field) by 3 (visual angle) ANOVA was performed on the forced-choice data. The responses to the pattern-match trials were evaluated separately. There was a main effect of visual angle

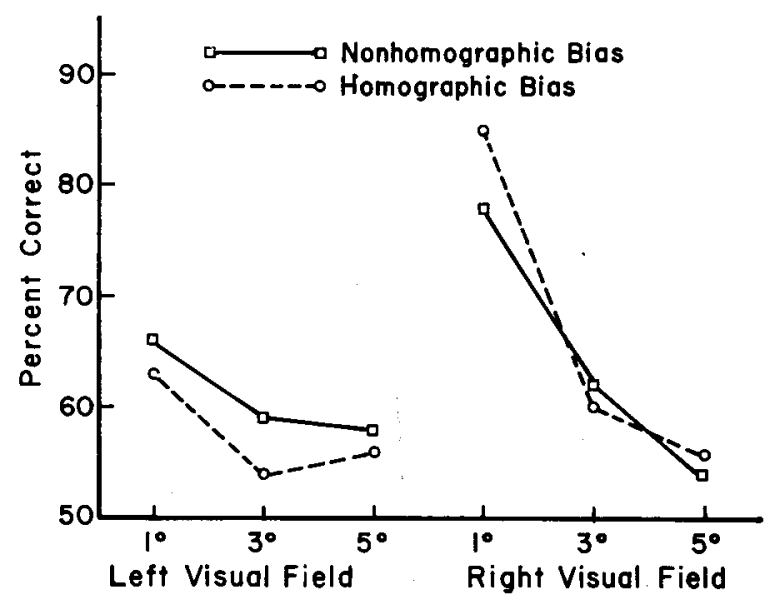

Figure 2. Percent correct choice dependent upon the location of the biasing word. 
$[F(2,34)=16.81, p<.001]$ and the effect for visual field approached significance $[\mathrm{F}(1,17)=3,84$, $\mathrm{p}<.06]$. Similar to the report analysis, homographic and nonhomographic bias did not differ significantly $(F<1)$ and visual angle interacted with visual field $[F(2,34)=4.45, p<.01]$. A Newman-Keuls test on the effect of visual angle indicated that the 1-deg position differed from the 3- and 5-deg positions, which did not differ significantly from each other. A test of simple effects on visual angle, dependent on visual field, resulted in differences for all visual angles to the right, while the pattern of results for visual angle to the left replicated the results of the Newman-Keuls test. There was also a significant effect of phrase location $[F(1,17)=29.78, p<.001]$ : that is, subjects tended to be more often correct for the upper phrase position. The separate evaluation of the pattern-match condition showed that, for trials in which no biasing information was parafoveally available, the probability of choosing the upper phrase was .6. This differential probability of choosing either phrase was assumed to indicate response bias. Hence, the observed effect of phrase location in the biased condition seems to be due to a tendency to choose the upper phrase when the biasing parafoveal word was not identified. A separate analysis in which the response bias was subtracted from the experimental manipulation yielded results identical to those described above. Thus, the resulting increase in correct choice for the upper phrase was counterbalanced by a corresponding decrease in correct choice for the lower phrase position.

\section{The Relationship Between Report and Forced-Choice Data}

Report and forced-choice data were related to shed light on the issue of unconscious semantic preprocessing of parafoveal words. It was expected that semantic preprocessing supported the correct choice behavior in those instances in which the biasing parafoveal word had not been correctly reported. A conditional probability value of correct choice dependent on no correct report was calculated and was $\mathrm{P}($ correct choice $/$ no correct report $)=.525$.

There were 1,106 trials in which the parafoveal word was not correctly reported. Five hundred and eighty-one (581) correct choices were made. This value corresponds to the margin reported in Bradshaw's (1974) investigation. To test the effect, a 2 (visual field) by 2 (homographic and nonhomographic bias) by 2 (correct and incorrect choice) ANOVA was performed on the forced-choice data.' Like Bradshaw (1974), raw data were transformed to percentage scores separately for correct and incorrect choices and the scores were each converted to an arc sine transformation. The test revealed that none of the main effects approached significance, with $F<1$ for the effects of visual field and homographic vs. nonhomographic bias; the effect of correct vs. incorrect choice was $[F(1,15)=1.70, p>.20]$. None of the interactions involving choice performance approached significance. This demonstrates that subjects did not reliably preprocess semantic information from the parafoveal area in those trials in which the parafoveal word was not successfully identified. However, the possibility remains that, on some trials, the parafoveal word had been correctly identified but could not be retrieved during the report task.

A model was developed to predict correct choice performance from report data. It was hypothesized that for a correct choice to occur, the subject either reported the parafoveal word and made a correct choice or did not report the parafoveal word but guessed correctly in the forced-choice task. This relationship is expressed in the equation:

$$
\mathrm{P}(\mathrm{CC})=\mathrm{P}(\mathrm{R}) \times \mathrm{P}(\mathrm{CC} / \mathrm{R})+\mathrm{P}(\overline{\mathrm{R}}) \times \mathrm{P}(\mathrm{CG}),
$$

with $\mathrm{CC}=$ correct forced choice, $\mathrm{R}=$ correct report (identification), $\bar{R}=$ no correct report, and $C G=$ correct guess in the forced-choice task. $P(R)$ and $P(C C / R)$ values were available for each of the different visual angles in both visual fields; $\mathrm{P}(\mathrm{CG})$ was set to be .5 . Thus,

$$
\mathrm{P}(\mathrm{CC})=\mathrm{P}(\mathrm{R}) \times \mathrm{P}(\mathrm{CC} / \mathrm{R})+[1-\mathrm{P}(\mathrm{R})] .5 .
$$

The predicted and observed values are given in Table 2 . The results show that, averaged across visual angle and visual field, predicted performance was $61 \%$ and observed performance was $63 \%$. This demonstrates that the forced-choice data can be explained by solely two factors: accomplished parafoveal word identification and correct guessing in the forced-choice task.

\section{Reaction Time for Forced Choice}

A correlation between forced-choice performance

Table 2

The Relation Between Predicted and Observed Correct Choice Performance

\begin{tabular}{lccccccc}
\hline & \multicolumn{4}{c}{ Visual Angle (in Degrees) } \\
\cline { 2 - 4 } & \multicolumn{3}{c}{ Left Visual Field } & & \multicolumn{3}{c}{ Right Visual Field } \\
\cline { 2 - 4 } \cline { 5 - 7 } & 1 & 3 & 5 & & 1 & 3 & 5 \\
\hline P(R) & .38 & .14 & .04 & .710 & .32 & .090 \\
P(CC/R) & .87 & .93 & .92 & .880 & .85 & .960 \\
$(1-$ R)G & .31 & .43 & .48 & .145 & .34 & .455 \\
P(CC) Predicted & .64 & .56 & .52 & .770 & .61 & .540 \\
P(CC) Observed & .65 & .56 & .55 & .810 & .61 & .570 \\
\hline
\end{tabular}

Note-These data are all based on the 16 subjects' performance where report and choice data were available. Only data from the biased conditions were included in this analysis. 
and the corresponding RTs was calculated, with a positive value indicating a higher degree of correct performance with increased RT. The correlation, averaged across subjects, was $r=.07$. The absence of a speed-accuracy tradeoff allowed for an independent evaluation of the choice and RT data. Two separate analyses of variance were performed on the RT data. First, the effects of semantic preprocessing in parafoveal vision on RT were evaluated; second, we determined whether the RT data were a sensitive indicator of semantic evaluation.

It was hypothesized that, even though semantic preprocessing had a negligible effect on choice performance, an effect could be revealed in the time to make a correct or an incorrect choice. To test this, a 2 (visual field) by 2 (homographic vs. nonhomographic bias) by 2 (correct or incorrect choice) ANOVA was performed on subjects' RT data for those trials in which the parafoveal word was not correctly reported. The statistical test indicated that none of the main effects approached significance, with $F<1$ in all cases. Also, an $\mathrm{F}<1$ value was obtained for all interactions involving choice performance. This result is consistent with the data analysis performed on the corresponding forced-choice data and constitutes an independent source of evidence against semantic preprocessing of words in parafoveal vision.

To test whether overall RT data were a valid indicator of semantic processing, a 2 (homographic and nonhomographic foveal word) by 3 (type of choice: correct, wrong, or neutral for the patternmatch trials) ANOVA was performed on the RT

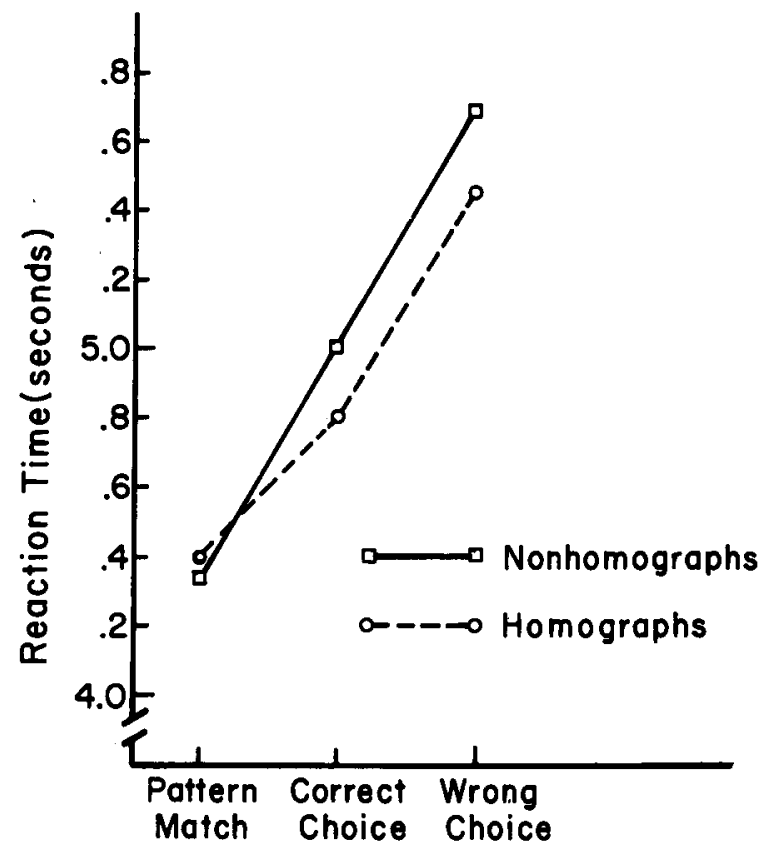

Figure 3. Reaction time for pattern match, correct, and incorrect choices in the forced-choice task. data (see Figure 3). A significant effect was obtained for type of choice $[F(2,30)=7.41, p<.005]$; the effect of ambiguity was not significant $(F<1)$. A Newman-Keuls test demonstrated that each of the three paired comparisons of type of choice resulted in a significant difference $(p<.01)$. This result indicates that the subject performed a semantic evaluation in order to decide which phrase to choose. That is, an incorrect choice is more likely to be the result of guessing between two possible forced-choice alternatives; hence, both forced-choice phrases had to be evaluated, which may have led to increased RT values. A correct choice is more likely to be the result of parafoveal word identification; hence, there was a higher probability that only one forced-choice alternative had to be evaluated in order to make a choice. The pattern match trials did not require any phrase evaluation and this way may have resulted in shortest $R T$ values.

Finally, a correlation between word frequency of the parafoveal word and choice behavior was calculated. It was expected that more frequent words should be identified or guessed correctly more often and, hence, lead to better forced-choice performance. However, this correlation was $r=.125$ for the homographic trials and $r=.005$ for the nonhomographic controls.

\section{DISCUSSION}

The results of this study are consistent with research reported by Rayner and Bertera (1979) and Rayner, McConkie, and Zola (1980); the concept of semantic preprocessing of parafoveal information was not supported. Semantic preprocessing was expected to affect performance in a forced-choice task, especially in those trials in which the subject failed to identify the critical parafoveal word. Contrary to this, the data showed that correct choice performance was not better than chance for those trials in which the parafoveal information was not correctly reported, and a comparison of report and forcedchoice data revealed that solely two factors (i.e., correct guessing in the forced-choice task and accomplished parafoveal word identification) were sufficient to explain the correct choice behavior. Thus, there was no evidence for effective semantic preprocessing. The evaluation of the error and partial report responses for the parafoveal word also confirm these results. Errors indicating semantic preprocessing were rare and may have been the result of chance factors (Ellis \& Marshall, 1978). An independent evaluation of the RT data supported this conclusion.

Although there was no demonstration of semantic preprocessing, there is evidence that visual information of a parafoveal word is available before the 
meaning is known. The low RT values for mere pattern-match trials, the absence of confusion errors, and the evaluation of incorrect and partial report responses of the parafoveal word support this conclusion. However, though visual information is available, it is not exploited in order to generate semantic expectations. This finding is similar to data, reported by Rayner and Bertera (1979), which showed that subjects who did not correctly identify the parafoveal word reported visually similar words.

Correct report and forced-choice performance also indicate that the reader can acquire sufficient information during the time frame of a single fixation to identify and evaluate more than a single word. This finding is qualified by characteristics of the visual angle and visual field information. The data show that the parafovea is not a homogeneous area; rather, parafoveal word identification is increasingly hampered the farther a word is projected from fixation position. This corresponds to a sharp decrease in visual acuity the farther an object is projected into the parafovea and the periphery; for example, pattern acuity has dropped $50 \%$ for an object located $1 \mathrm{deg}$ of visual angle from the center of the fovea, and, for words $8 \mathrm{deg}$ from the center, it is only $15 \%$ of the maximum (Riggs, 1965). This seems to suggest that the physiological structure of the visual system is a determinant of the ability to identify a nonfoveally presented word.

It is the importance of retinal position and visual field factors which does not allow us to extrapolate the present findings to a dichotic listening situation. Lackner and Garrett (1972) and Lewis (1970) showed that listeners were influenced by semantic information presented in the unattended channel, although they were unable to report the unattended words or sentences. However, in a dichotic listening situation, both messages can be administered to two different receptor systems which are anatomically and functionally identical. Thus, it remains possible that semantic preprocessing may operate in auditory perception.

\section{CONCLUSION}

The present data do not support a model of word perception which relies on semantic preprocessing of parafoveal information. Rather, semantic information can be obtained from the parafoveal area, but this information is directly available and is not contingent upon a previous unconscious semantic representation. In accordance with this finding, a processmonitoring model states that the reader gains semantic information only for the words identified. It is further assumed in this model that the reader tries to identify words as far into the right of a line of text as possible during an individual fixation, which implies that in many fixations more than one word will be semantically evaluated. Adams (1979) and Rayner, Inhoff, Morrison, Slowiaczek, and Bertera (in press) report results which are consistent with this position. Their data show that sufficient visual information can be extracted from a foveally presented word within $50 \mathrm{msec}$, so that the word becomes reportable. This may leave the remainder of fixation duration for additional evaluation of parafoveal words.

\section{REFERENCES}

Adams, M. J. Models of word recognition. Cognitive Psychology, 1979, 11, 133-176.

Allport, D. A. On knowing the meaning of words we are unable to report: The effects of visual masking. In S. Dornic (Ed.), Attention and performance. VI. Hillsdale, N.J: Erlbaum, 1977.

Bradshaw, J. L. Peripherally presented and unreported words may bias the perceived meaning of a centrally fixated homograph. Journal of Experimental Psychology, 1974, 103, 1200-1202.

Cramer, P. A study of homographs. In L. Postman \& G. Keppel (Eds.), Norms and word associations. New York: Academic Press, 1970.

Ellis, A. W., \& Marshall, J. C. Semantic errors or statistical flukes? A note on Allport's "On knowing the meaning of words we are unable to report: The effects of visual masking." Quarterly Journal of Experimental Psychology, 1978, 30, 569-575.

Hoch8e RG, J. Components of literacy: Speculations and exploratory research. In $\mathbf{H}$. Levin \& J. P. Williams (Eds.), Basic studies on reading. New York: Basic Books, 1970.

HUEY, E. B. The psychology and pedagogy of reading. New York: Macmillan, 1908. (Republished by M.I.T. Press, 1977.)

KuČera. H., \& Francis, W. N. Computational analysis of present day American English. Providence, R.I: Brown University Press, 1967.

Lackner, J. R., \& Garrett, M. F. Resolving ambiguity: Effects of biasing context in the unattended ear. Cognition, 1972, 1/4, 359-373.

LEwIS, J. L. Semantic processing of unattended messages using dichotic listening. Journal of Experimental Psychology, 1970, 85, 225-228.

MARCEL, T. Unconscious reading: Experiments on people who do not know they are reading. Visible Language, 1978, 12, 392-404.

McConkie, G. W. On the role and control of eye movements in reading. In P. A. Kolers, M. E. Wrolstad, \& H. Bouma (Eds.), Processing of visual language (Vol. 1). New York: Plenum Press, 1979.

McConkie, G. W., \& Rayner, K. The span of the effective stimulus during a fixation in reading. Perception \& Psychophysics, 1975, 17, 578-586.

Nersser, U. Cognitive psychology. New York: Appleton-CenturyCrofts, 1967.

RAYNER, $K$. The perceptual span and peripheral cues in reading. Cognitive Psychology, 1975, 7, 65-81.

$R_{A Y N E R}, K$. Eye movements in reading and information processing. Psychological Bulletin, 1978, 85, 618-660.

RAYNER, K. Eye movements in reading: Eye guidance and integration. In P. A. Kolers, M. E. Wrolstad, \& H. Bouma (Eds.), Processing of visual language (Vol. 1). New York: Plenum Press, 1979.

Rayner, K., \& Bertera, J. Reading without a fovea. Science, 1979, 206, 468-469.

Rayner, K., McConkie, G. W., \& Zola, G. S. Integrating 
information across eye movements. Cognitive Psychology, 1980, 12, 206-226.

Rayner, K., Inhoff, A. W., Morrison, R. E., Slowiaczek, M., \& BERTERA, J. Masking of foveal and parafoveal vision during eye fixations in reading. Journal of Experimental Psychology: Human Perception and Performance, in press.

Riggs, L. A. Visual acuity. In C. H. Graham (Ed.), Vision and visual perception. New York: Wiley, 1965.

Websters New Collegiate Dictionary. Springfield, Mass: Merriam, 1973.

\section{NOTE}

1. The effect of visual angle was not included in the analysis. The low number of instances allowing for semantic preprocessing at the 1-deg-of-visual-angle position due to the high report did not allow for a reliable mean estimation per subject.

(Received for publication November 8, 1979; revision accepted February $18,1980$. ) 